\title{
New SPECT technology: potential and challenges
}

\author{
Brian F. Hutton
}

Published online: 20 July 2010

(C) Springer-Verlag 2010

\section{Potential}

Major advances in organ-specific imaging

The article by Bocher et al. in this issue [1] reports on the performance of a novel SPECT system specifically designed for cardiac imaging based on the use of a set of pinhole collimators in combination with cadmium zinc telluride (CZT) detectors. The system is inspired by earlier work on multipinhole imaging [2] and the conceptual design has previously been demonstrated in simulation studies [3]. Also early clinical validation studies have appeared recently $[4,5]$. The described system (available from GE Healthcare) joins a range of novel organ-specific commercial systems that have recently been introduced to the consumer with reported improvement in resolution and sensitivity (usually reported as potential for reduced acquisition time; see recent reviews $[6,7])$. Specific examples are the D-SPECT from Spectrum Dynamics [8,9] and the Cardius from Digirad [10]. Clinical validation studies for these systems are now also appearing (e.g. [11, 12]). The developers of these systems deserve congratulations for introducing innovations that undoubtedly yield the largest advance in SPECT performance since the introduction of the rotating gamma camera, which has dominated clinical use for several decades. Until recently the development in SPECT performance has been incremental, mainly involving refinement rather than major changes in system design, perhaps mainly due to the clinical demand for systems that offer flexibility in application. The recent innovation has been inspired at least partly by the rapid

B. F. Hutton $(\triangle)$

Institute of Nuclear Medicine, University College London,

235 Euston Road,

London, NWI 2BU, UK

e-mail: brian.hutton@uclh.nhs.uk developments in preclinical imaging systems where ultrahigh resolution and relatively high sensitivity have been demonstrated. Bringing new designs to fruition has only been possible due to the combination of several factors: the improved reliability of alternative detector materials, the development of fast electronics, the development of efficient reconstruction algorithms and, not least, the demand for organ-specific systems where optimization for a specific purpose rather than flexibility in application is indicated.

\section{CZT: pros and cons}

Central to the design of both the GE Healthcare system and the D-SPECT is the use of CZT. Although not a new detector material, it is only recently that the reliability and performance of CZT at room temperature have been sufficiently improved to make clinical use a possibility. The cost of CZT remains relatively high and places a constraint on the possible designs. But the appeal is not just the much improved energy resolution (typically 5-6\%) but particularly the small size that facilitates novel compact designs, which are very much appealing for use in organspecific imaging. There are alternative approaches to achieving compact designs based on conventional scintillation detectors coupled to optical readout systems other than conventional photomultiplier tubes (e.g. avalanche photodiode or silicon photomultiplier), which may have a cost advantage (see [13] for a useful overview). We can anticipate increasing use of new technology in future system designs for both SPECT and PET.

Innovative reconstruction

The importance of image reconstruction as a component of new technology should not be underestimated, especially 
the flexibility of iterative reconstruction techniques in adapting to novel detector geometry. There is increasing recognition of the value of including a complete system model that incorporates not only details of the emission process but also characteristics of the imaging system directly in the reconstruction algorithm. Historically the time required for more complex reconstruction was untenable, even with acceleration algorithms such as ordered subsets expectation maximization (OS-EM) [14]; however, this barrier no longer exists. As a result quite complex geometry and nonstationary effects (both spatially-variant resolution and angularly variable acquisition time) can be readily accommodated. Use of iterative reconstruction is therefore now standard practice, and in the case of new technology is normally the only option. The appeal in using iterative reconstruction is not only its flexibility but also its favourable noise properties.

\section{Extended applications}

The potential offered by these systems extends beyond the immediate appeal of reduced scanning time, which can be at least partly achieved using alternative approaches with imaginative collimators (e.g. Siemens Smartzoom collimator, a recent revision of cardiofocal collimation [15]) and/or reconstruction strategies (e.g. modelling the system spatial response $[16,17])$. Bocher et al. point out a wide range of potential applications for the new technology, mainly applications that are feasible with standard gamma cameras, but where the newer technology offers potential technical advantage. It remains to be seen how much advantage is achieved in implementing the potential range of applications in a clinical setting. Dynamic acquisition is normally hampered by limited statistics which is potentially improved in the new systems; the GE system has the added advantage of utilizing stationary detectors so that rapid frame-rates can be achieved without danger of introducing artefacts due to change of activity distribution during acquisition. Dynamic studies are currently being assessed as a means of determining flow and coronary reserve indices using SPECT tracers, as highlighted in a recent editorial [17]. The use of CZT provides potential for superior energy discrimination that may encourage increased use of simultaneous dual or multiple radionuclide studies; these not only provide efficient patient protocols but reduce the need for interstudy registration due to patient movement. The simultaneous use of ${ }^{99 \mathrm{~m}} \mathrm{Tc}$ and ${ }^{201} \mathrm{Tl}$ for simultaneous rest/stress myocardial perfusion imaging has been recently demonstrated for the D-SPECT system [18]. An advantage of the higher sensitivity systems is the ability to reduce the activity of the ${ }^{99 \mathrm{~m}} \mathrm{Tc}$-labelled tracer so as to reduce down-scatter to the lower energy photopeak. The new technology therefore plays an important role in encouraging the adoption of alternative protocols that were previously considered impractical. This suggests many exciting possibilities.

\section{Challenges}

\section{Clinical issues}

Clearly organ-specific systems can provide better performance than general systems, being specifically designed for the unique application. Of course there needs to be sufficient clinical demand for the specific application, and cardiac perfusion imaging currently does meet this requirement in many centres. This does, however, raise concerns regarding the long-term use of these systems as, unlike conventional cameras, they cannot be relegated to alternative tasks as the equipment ages or is replaced by newer technology. As with any change in technology there are inevitably changes in image appearance that the end-user must recognize and learn to interpret. For example, several of the cardiac-specific systems permit upright or semiupright acquisition with improved patient comfort but consequential changes in heart geometry that influence both the normal appearance and the nature of attenuation artefacts. The tendency is to always compare results with current practice rather than to investigate if the new technology provides enhanced diagnostic information. The dilemma is the need to utilize standard processing software which is specifically adapted to the older technology, but which may be substandard for use with newer technology. This places considerable pressure on the suppliers to simply demonstrate equivalence rather than superiority.

\section{System performance}

An area of some concern is the difficulty in adequately describing performance of novel SPECT systems, especially when these utilize iterative reconstruction. Clearly direct comparison with conventional SPECT system performance is desirable where specification is based on well-established NEMA protocols. The consumer has grown accustomed to the use of published figures on spatial resolution and sensitivity as an indication of achievable clinical performance, and these parameters permit meaningful crosscomparison across available conventional systems. It should be stressed, however, that NEMA protocols include not only exact description of the acquisition protocol but also a prescription for image reconstruction and subsequent processing; deviation from this protocol negates the validity of the measurements. The challenge is how to provide comparable specifications when using instead iterative reconstruction. Both resolution and noise are not only 
influenced by choice of reconstruction parameters but are also object-dependent. The following sections illustrate the problem and highlight the need for the introduction of revised standards that facilitate meaningful comparison of systems.

When is resolution not resolution?

NEMA specifications for resolution involve the use of filtered back projection using a ramp filter; clearly results would be meaningless if instead some arbitrary filter was applied. The situation is more complex for iterative reconstruction as resolution versus noise varies with iteration number in a complex way, with the rate of convergence dependent on the activity distribution. Figure 1 illustrates the variation in resolution with iteration number for an isolated point source in air (Air), a point source with low background activity $(0.1 \%)$ (lowbg) and a low activity point source (1.0\%) in a constant background activity (lowpt). In each case the data were reconstructed using OS-EM including a model of the system's spatial response. Resolution was estimated from the reconstructed point response function. The observed full-width at half-maximum that is ultimately reached is dramatically different in the three situations (2.25, 5.24 and 8.54 for air, lowbg and lowpt, respectively). Clearly the use of an isolated line or point source very much overstates the achievable resolution. This will be further complicated in clinical practice where there is likely to be a more complex activity distribution as was highlighted in the paper by Erlandsson et al. [9] in evaluation of the D-SPECT system. In this case attempts were made to estimate resolution using a clinically realistic activity distribution using a perturbation approach. This involved combining

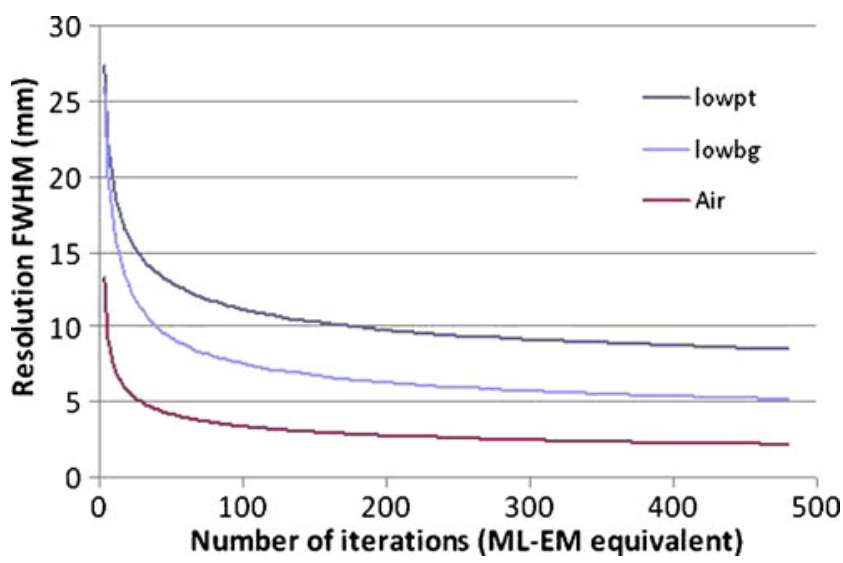

Fig. 1 Variation in resolution with iteration number for an isolated point source in air (Air), a point source with low background activity $(0.1 \%)($ lowbg) and a low activity point source $(1.0 \%)$ in a constant background activity (lowpt) measurements using a line source with measurements of the activity distribution in order to estimate the resolution that might be expected at a given location. A similar approach has been previously suggested using simulation [19-21]. The approach needs further evaluation and the protocol needs to be optimized; however, the approach at least attempts to demonstrate more realistic figures than those obtained for an isolated source. Caution therefore is needed when presented with figures for resolution derived from point or line source measurements reconstructed using iterative methods.

When is sensitivity not sensitivity?

In conventional systems the time required for the acquisition of a selected number of counts is determined by the system's sensitivity, with an expectation that the counts that can be acquired in a given time are sufficiently high so as to limit statistical noise in the acquired data and reconstructed images. The feasible reduction in acquisition time is therefore assumed to be inversely proportional to increased sensitivity. In practice, however, using iterative reconstruction the reconstructed noise will depend once again on the number of iterations as well as the complexity of reconstruction (or degree of uncertainty in the origin of each acquired photon). Increased counts are usually acquired at the expense of resolution; the worse the resolution the greater the uncertainty in localization. The consequence is that 'bad' counts contribute less towards the final signal, requiring more than the anticipated number of counts to achieve a targeted noise level. Similar issues can arise if there is overlap of data from multiple pinholes or slit apertures (so-called multiplexing). It cannot therefore be assumed that the stated sensitivity necessarily predicts the achievable noise gain or reduction in acquisition time. All quite confusing to the consumer who in the past has placed some trust in standard sensitivity specifications.

Need for updated standards

As with any new technology there is the need for consumers and industry to work together to define meaningful tests that can be performed to verify specification of equipment. These provide the basis for acceptance testing, but also tend to form the basis for quality control and meaningful comparison of systems. As system design becomes more diverse the design of standard tests becomes more challenging as standardizing protocols becomes difficult, if not impossible. In the meantime stated performance parameters need to be treated with some reservation. 


\section{Conclusion}

Novel SPECT technology for organ-specific imaging, such as the system described by Bocher et al., is a welcome addition to the nuclear medicine armoury and offers reduced acquisition time (or radiation dose) and, more importantly, the opportunity for extended application. Measurement of performance on systems whose designs demand iterative reconstruction is not straightforward as performance parameters are dependent on not only reconstruction parameters but also the activity distribution being imaged. The lack of standard performance measurement protocols relevant to the new technology makes meaningful cross-comparison difficult. Despite these limitations it is clear that the recent advances in technology result in SPECT performance that is superior to that of conventional rotating dual-detector Anger technology.

Acknowledgments Thanks to Kjell Erlandsson for assistance with the figure included in this article. The author is in receipt of research support from both GE Healthcare and Spectrum Dynamics. Also UCL/UCLH receives a portion of its research support from the UK Department of Health's NIHR Biomedical Research Centres funding scheme.

\section{References}

1. Bocher M, Blevis I, Tsukerman L, Shrem Y, Kovalski G, Volokh L. A fast cardiac gamma camera with dynamic SPECT capabilities: design, system validation and future potential. Eur J Nucl Med Mol Imaging 2010;37. doi:10.1007/s00259-010-1488-Z.

2. Funk T, Kirch DL, Koss JE, Botvinick E, Hasegawa BH. A novel approach to multipinhole SPECT for myocardial perfusion imaging. J Nucl Med 2006;47:595-602.

3. Huang Q, Boutchko, Hasegawa R, Budinger BH, Gullberg TF, Grant T. Computer evaluation of a novel multipinhole SPECT system for cardiac imaging. Nuclear Science Symposium Conference Record, 2008. NSS '08. IEEE. p. 4025-7. doi:10.1109/ NSSMIC.2008.4774167

4. Patton JA, Slomka PJ, Germano G, Berman DS. Recent technologic advances in nuclear cardiology. J Nucl Cardiol 2007;14:501-13.

5. Slomka PJ, Patton JA, Berman DS, Germano G. Advances in technical aspects of myocardial perfusion SPECT imaging. J Nucl Cardiol 2009;16:255-76.

6. Esteves FP, Raggi P, Folks RD, et al. Novel solid-state-detector dedicated cardiac camera for fast myocardial perfusion imaging: multicenter comparison with standard dual detector cameras. J Nucl Cardiol 2009;16:927-34.

7. Buechel RR, Herzog BA, Husmann L, et al. Ultrafast nuclear myocardial perfusion imaging on a new gamma camera with semiconductor detector technique: first clinical validation. Eur J Nucl Med Mol Imaging 2010;37:773-8.

8. Gambhir SS, Berman DS, Ziffer J, Nagler M, Sandler M, Patton J, et al. A novel high-sensitivity rapid-acquisition single-photon cardiac imaging camera. J Nucl Med 2009;50:635-43.

9. Erlandsson K, Kacperski K, van Gramberg D, Hutton BF, Performance evaluation of D-SPECT: a novel SPECT system for nuclear cardiology. Phys Med Biol 2009;54:2635-49.

10. Bai C, Conwell R, Kindem J, Babla H, Gurley M, De Los Santos $\mathrm{R}$, et al. Phantom evaluation of a cardiac SPECT/VCT system that uses a common set of solid-state detectors for both emission and transmission scans. J Nucl Cardiol 2010;17:459-69.

11. Sharir T, Ben-Haim S, Merzon K, Prochorov V, Dickman D, Berman DS. High speed myocardial perfusion imaging initial clinical comparison with conventional dual detector anger camera imaging. JACC Cardiovasc Imaging 2008;1:156-63.

12. Sharir T, Slomka PJ, Hayes SW, DiCarli MF, Ziffer JA, Martin $\mathrm{WH}$, et al. Multicenter trial of high-speed versus conventional single-photon emission computed tomography imaging: quantitative results of myocardial perfusion and left ventricular function. J Am Coll Cardiol 2010;55:1965-74.

13. Pichler BJ, Ziegler SI. Photodetectors. In: Wernick MN, Aarsvold JN, editors. Emission tomography: the fundamentals of SPECT and PET. San Diego: Elsevier; 2004. p. 270-92.

14. Hudson HM, Larkin RS. Accelerated image reconstruction using ordered subsets of projection data. IEEE Trans Med Imaging 1994;13:601-9.

15. Hawman P, Haines EJ. The cardiofocal collimator: a variable focus collimator for cardiac SPECT. Phys Med Biol 1994;39:439 50.

16. Ali I, Ruddy TD, Almgrahi A, Anstett FG, Wells RG. Half-time SPECT myocardial perfusion imaging with attenuation correction. J Nucl Med 2009;50:554-62.

17. Cuocolo A, Petretta M, Soricelli A. Measurement of coronary flow reserve by noninvasive cardiac imaging. Eur J Nucl Med Mol Imaging 2919;37:1198-202.

18. Ben-Haim S, Kacperski K, Hain S, Van Gramberg D, Hutton BF, Erlandsson K, et al. Simultaneous dual-radionuclide myocardial perfusion imaging with a solid-state dedicated cardiac camera. Eur J Nucl Med Mol Imaging 2010;37(9). doi:10.1007/s00259-0101441-1.

19. Stamos JA, Rogers WL, Clinthorne NH, Koral KF. Object dependent performance comparison of two iterative reconstruction algorithms. IEEE Trans Nucl Sci 1988;35:611-4.

20. Liow JS, Strother SC. The convergence of object dependent resolution in maximum likelihood based tomographic image reconstruction. Phys Med Biol 1993;38:55-70.

21. Du Y, Tsui BMW, Frey EC. Partial volume effect compensation for quantitative brain SPECT imaging. IEEE Trans Med Imaging 2005;24:969-76. 